\title{
DIFFERENTIABLE FUNCTIONS DEFINED IN CLOSED SETS. I $\dagger$
}

\author{
BY \\ HASSLER WHITNEY
}

1. Introduction. In a recent paper§ the author has shown that if a function $f(x)$ defined in a closed set $A$ in $n$-space $E$ satisfies certain conditions involving Taylor's formula (in finite form), i.e. if it is "of class $C^{m}$ in $A$," then its definition can be extended over $E$ so that it will have continuous partial derivatives through the $m$ th order. In this paper we restrict ourselves to the one-dimensional case. (For the above theorem in this case, see $\$ 4$.) Let $x_{0}, \cdots, x_{m}$ be distinct points of $A$. If $P(x)=c_{0}+\cdots+c_{m} x^{m}$ is the polynomial of degree at most $m$ such that $P\left(x_{i}\right)=f\left(x_{i}\right)(i=0, \cdots, m)$, the $m$ th difference quotient of $f(x)$ at these points is $\Delta_{0} \ldots m f=\Delta^{m} f(x)=m ! c_{m}$. The main object of this paper is to prove (see $\$ \S 2$ and 3 for definitions)

Theorem I. A necessary and sufficient condition that $f(x)$ be of class $C^{m}$ in $A$ is that $\Delta^{m} f(x)$ converge in $A$.

This theorem furnishes a direct definition of the differentiability of a function; the former definition (see $\S 3$ ) involved the existence of other functions $f_{1}(x), \cdots, f_{m}(x)$.

The necessity of the condition is easily proved. The definition of $f(x)$ being extended over the $x$-axis $E$, consider any $m+1$ points $x_{0}, \cdots, x_{m}$ $\left(x_{0}<x_{1}<\cdots<x_{m}\right)$. Define $P(x)$ as above. As $f\left(x_{i}\right)-P\left(x_{i}\right)=0(i=0, \cdots, m)$ there is a point $x^{\prime}\left(x_{0}<x^{\prime}<x_{m}\right)$ such that $\left(d^{m} / d x^{m}\right)\left[f\left(x^{\prime}\right)-P\left(x^{\prime}\right)\right]=0$. But $d^{m} P(x) / d x^{m} \equiv m ! c_{m}=\Delta_{0} \ldots{ }_{m} f$; hence $\Delta_{0} \ldots{ }_{m} f=d^{m} f\left(x^{\prime}\right) / d x^{m}$. Therefore if $x_{0}, \cdots, x_{m}$ are in $A$ and are sufficiently near a point $x^{*}$ of $A, \Delta_{0} \ldots m_{m} f$ $=d^{m} f\left(x^{\prime}\right) / d x^{m}=d^{m} f\left(x^{*}\right) / d x^{m}$ approximately, and $\Delta^{m} f(x)$ converges in $A$ (in fact, in $E$ ). This may be proved also from (2.6) for $s=m$.

We note that, for $f(x)=f_{0}(x)$ to be of class $C^{m}$ in a general closed set $A$, it is not sufficient that there exist functions $f_{s}(x)(s=1, \cdots, m)$ in $A$ such that $d f_{s}(x) / d x=f_{s+1}(x)$ there. As an example, set $f_{0}(0)=0$ and $f_{0}(x)=1 / 2^{2 i}$ $\left(1 / 2^{i} \leqq x \leqq 3 / 2^{i+1}, i=1,2, \cdots\right)$, and set $f_{1}(x) \equiv 0$ and $f_{2}(x) \equiv 0$ in the same point set $A$.

The majority of the paper is devoted to the proof of Theorem I. In the

$\dagger$ Presented to the Society, October 28, 1933; received by the editors July 27, 1933.

‡ National Research Fellow.

$\$$ Analytic extensions of differentiable functions defined in closed sets, these Transactions, vol. 36 (1934), pp. 63-89; this paper will be referred to as A.E. 
last section we study Taylor's formula in finite form, when it holds in closed sets, and when its validity implies differentiability of the given function.

2. Difference quotients. $\dagger$ If $x_{0}, \cdots, x_{m}$ are distinct numbers, set $\ddagger$

$$
u_{i j}=x_{i}-x_{i}, \quad r_{i j}=\left|u_{i j}\right|, \quad \alpha_{01}^{i} \cdots m=\frac{1}{u_{0 i} \cdots u_{i-1, i} u_{i+1, i} \cdots u_{m i}}
$$

Given a function $f(x)$, we define the $m$ th difference quotient by the formula

$$
\Delta^{m} f(x)=\Delta\left(x_{0}, x_{1}, \cdots, x_{m} ; f\right)=\Delta_{01} \ldots m f=m ! \sum_{i=0}^{m} \alpha_{01}^{i} \ldots m f\left(x_{i}\right) .
$$

In particular, $\Delta_{0} f=f\left(x_{0}\right), \Delta_{01} f=\left[f\left(x_{1}\right)-f\left(x_{0}\right)\right] /\left(x_{1}-x_{0}\right) . \Delta_{0} \ldots m$ is symmetric in the points $x_{0}, \cdots, x_{m}$.

If $i \geqq 2$,

$$
\frac{1}{u_{01}}\left(\alpha_{12 \ldots,}^{i}-\alpha_{02}^{i} \ldots s\right)=\frac{1}{u_{01}}\left(\frac{1}{u_{1 i} \cdots}-\frac{1}{u_{0 i} \cdots}\right)=\frac{1}{u_{01}} \frac{u_{0 i}-u_{1 i}}{u_{0 i} u_{1 i} \cdots}=\alpha_{012 \ldots s}^{i} ;
$$

hence

$$
\begin{aligned}
\frac{s}{u_{01}}\left(\Delta_{12} \ldots s-\Delta_{02} \ldots s\right)= & \frac{s !}{u_{01}}\left[-\alpha_{02}^{0} \ldots s f\left(x_{0}\right)+\alpha_{12}^{1} \ldots s f\left(x_{1}\right)\right. \\
& \left.+\sum_{i \geq 2}\left(\alpha_{12}^{i} \ldots s-\alpha_{02}^{i} \ldots s\right) f\left(x_{i}\right)\right] \\
= & s ! \sum_{i=0}^{s} \alpha_{012}^{i} \ldots s f\left(x_{i}\right)=\Delta_{012} \ldots s .
\end{aligned}
$$

Suppose $*$ is a set of subscripts containing neither 0,1 , nor 2 ; then for some $m$,

$$
\Delta_{012 *}=\frac{m}{u_{01}}\left(\Delta_{12 *}-\Delta_{02 *}\right)=\frac{m}{u_{02}}\left(\Delta_{12 *}-\Delta_{01 *}\right) .
$$

Solving for $\Delta_{01 *}$, we find

$$
\Delta_{01 *}=\frac{u_{02}}{u_{01}} \Delta_{02 *}+\frac{u_{21}}{u_{01}} \Delta_{21 *}
$$

which may be written as follows: $u_{01} \Delta_{01 *}+u_{12} \Delta_{12 *}+u_{20} \Delta_{20 *}=0$.

Let $x_{0}, \cdots, x_{s}$ be distinct numbers. If we solve the equations $\sum_{i=0}^{i}$

† Compare Nörlund, Differenzenrechnung, Berlin, 1924, pp. 8-9. It is seen that $\Delta_{01} \ldots{ }_{m}=m$ ! $\left[x_{0} x_{1} \cdots x_{m}\right]$.

$\ddagger$ In the equations below, the numbers $0,1, \cdots$, when appearing as subscripts, are to be considered as variables. Thus, as a particular case of $(2.1), \alpha_{023}^{0}=1 /\left(u_{20} u_{30}\right)$; in the second equation of $\$ 6$, $\Sigma_{j} 1 / u_{j^{\prime} i}=1 / u_{0^{\prime} i}+\cdots$. Without this notation, the equations would often get quite cumbersome. 
$\left(x_{i}-x\right)^{i} z_{i}=\delta_{j \bullet}(j=0, \cdots, s), x$ being any fixed number, we find $z_{i}=\alpha_{0}^{i} \cdots$. Hence

$$
\begin{aligned}
& \sum_{i=0}^{s} \alpha_{0}^{i} \ldots s\left(x_{i}-x\right)^{i}=0 \quad(j=0, \cdots, s-1), \\
& \sum_{i=0}^{\dot{2}} \alpha_{0}^{i} \ldots_{8}\left(x_{i}-x\right)^{*}=1 .
\end{aligned}
$$

Suppose $f(x)=f_{0}(x), \cdots, f_{m}(x), R\left(x^{\prime}, x\right)=R_{0}\left(x^{\prime}, x\right)$ satisfy (3.1) below for $s=0$. Then (2.2) and (2.5) give

$$
\begin{aligned}
\Delta_{0} \ldots, f & =s ! \sum_{i=0}^{s} \alpha_{0}^{i} \ldots,\left[\sum_{j=0}^{m} \frac{f_{j}(x)}{j !}\left(x_{i}-x\right)^{i}+R\left(x_{i}, x\right)\right] \\
& =f_{\bullet}(x)+s ! \sum_{i=s+1}^{m} \frac{f_{j}(x)}{j !} \sum_{i=0}^{\dot{1}} \alpha_{0}^{i} \ldots,\left(x_{i}-x\right)^{i}+s ! \sum_{i=0}^{\dot{1}} \alpha_{0}^{i} \ldots, R\left(x_{i}, x\right) .
\end{aligned}
$$

If $f(x)=c_{0}+\cdots+c_{m} x^{m}$ is a polynomial of degree at most $m$, then (3.1) is satisfied with $f_{m}(x) \equiv m ! c_{m}$ and $R_{s}\left(x^{\prime}, x\right) \equiv 0$. Setting $s=m$ in (2.6) gives

$$
\Delta_{0} \ldots m f \equiv m ! c_{m} .
$$

We say $\Delta^{m} f(x)$ converges in the set $A$ if for. each point $x$ of $A$ and every $\epsilon>0$ there is a $\delta>0$ such that if $x_{0}, \cdots, x_{m}, x_{0^{\prime}}, \cdots, x_{m^{\prime}}$ are any two sets of distinct points of $A$, all within $\delta$ of $x$, then

$$
\left|\Delta_{0} \ldots m-\Delta_{0} \cdots m^{\prime}\right|<\epsilon .
$$

$\Delta^{m} f(x)$ of course converges at all isolated points of $A$. We say $\Delta^{m} f(x) \rightarrow f_{m}(x)$ in $A$ if $\left|\Delta_{0} \ldots m-f_{m}(x)\right|<\epsilon$ whenever $x_{0}, \cdots, x_{m}$ are in $A$ and within $\delta$ of $x$. Evidently if $\Delta^{m} f(x) \rightarrow f_{m}(x)$ in $A$, then $f_{m}(x)$ is continuous in the set of limit points of $A$.

\section{DIFFERENTIABLE FUNCTIONS}

3. Definition of differentiable functions. Let $f(x)=f_{0}(x)$ be defined in the closed set $A$. We say $f(x)$ is of class $C^{m}$ in $A$ (see A. E.) if there exist functions $f_{1}(x), \cdots, f_{m}(x), R\left(x^{\prime}, x\right)=R_{0}\left(x^{\prime}, x\right), \cdots, R_{m}\left(x^{\prime}, x\right)$ in $A$ such that

$$
f_{\imath}\left(x^{\prime}\right)=\sum_{i=s}^{m} \frac{f_{i}(x)}{(i-s) !}\left(x^{\prime}-x\right)^{i-\imath}+R_{s}\left(x^{\prime}, x\right) \quad(s=0, \cdots, m),
$$

and for each $s$, each point $x$ of $A$, and every $\epsilon>0$ there is a $\delta>0$ such that

$$
\left|\frac{R_{0}\left(x^{\prime \prime}, x^{\prime}\right)}{\left(x^{\prime \prime}-x^{\prime}\right)^{m-\bullet}}\right|<\epsilon \quad\left(x^{\prime}, x^{\prime \prime} \text { in } A ;\left|x^{\prime}-x\right|,\left|x^{\prime \prime}-x\right|<\delta\right) .
$$

If $f_{i}(x), \cdots, f_{m}(x), R_{i}\left(x^{\prime}, x\right)$ satisfy (3.1) and (3.2) for $s=i$, we say $f_{i}(x)$ 
can be expanded in a Taylor's formula to the $(m-i)$ th order locally uniformly in terms of $f_{i}(x), \cdots, f_{m}(x)$. If $f(x)$ is defined throughout an open interval and has a continuous $m$ th derivative there, then it is of class $C^{m}$, by Taylor's theorem.

4. Extension of differentiable functions. If $f_{0}(x)$ is of class $C^{m}$ in terms of $f_{0}(x), \cdots, f_{m}(x)$ in $A$, then the definitions of these functions can be extended throughout $E$ so they will be continuous and so that $d f_{s}(x) / d x=f_{s+1}(x)$ there $(s=0, \cdots, m-1)$ (see A. E., Lemma 2). As the proof can be given more simply in the one-dimensional case, we give it here. We can assume $A$ is unbounded on both sides; otherwise, take a point $a$ beyond $A$ on either side, and set $f_{s}(x) \equiv 0(s=0, \cdots, m)$ beyond $a$.

For each interval $(a, b)$ of $E-A$, let $P(x)$ be the polynomial of degree at most $2 m+1$ such that

$$
\frac{d^{e}}{d x^{s}} P(a)=f_{s}(a), \quad \frac{d^{s}}{d x^{s}} P(b)=f_{s}(b) \quad(s=0, \cdots, m) ;
$$

we set

$$
f_{s}(x)=\frac{d^{\bullet}}{d x^{s}} P(x) \text { in }(a, b) .
$$

$d f_{s}(x) / d x=f_{s+1}(x)(s=0, \cdots, m-1)$ in $E-A$; we must show that this holds also at any point $x_{0}$ of $A$.

Suppose each $f_{s+1}(x)$ is continuous in $E$. Then given $x_{0}$ in $A$ and $\epsilon>0$, take $\delta>0$ so small that

$$
\left|f_{s+1}\left(x^{\prime}\right)-f_{s+1}\left(x_{0}\right)\right|<\frac{\epsilon}{2} \quad\left(\left|x^{\prime}-x_{0}\right|<\delta\right) .
$$

By (3.1) and (3.2), we can also take $\delta$ so small that if $a$ is in $A,\left|a-x_{0}\right|<\delta$, and

$$
f_{s}(a)=f_{s}\left(x_{0}\right)+f_{s+1}\left(x_{0}\right)\left(a-x_{0}\right)+R^{\prime}\left(a, x_{0}\right),
$$

then $\left|R^{\prime}\left(a, x_{0}\right) /\left(a-x_{0}\right)\right|<\epsilon / 2$. Now take any point $x$ within $\delta$ of $x_{0}$. If $x$ is in $A$, set $a=x$; otherwise, let $a$ be the end point nearest $x_{0}$ of the interval of $E-A$ containing $x$. Now for some $x^{\prime}, a \leqq x^{\prime} \leqq x$,

$$
f_{s}(x)=f_{s}(a)+f_{s+1}\left(x^{\prime}\right)(x-a) .
$$

Adding this to the last equation and dividing by $x-x_{0}$, we find

$$
\frac{f_{s}(x)-f_{s}\left(x_{0}\right)}{x-x_{0}}=f_{s+1}\left(x_{0}\right)+\left[f_{s+1}\left(x^{\prime}\right)-f_{s+1}\left(x_{0}\right)\right] \frac{x-a}{x-x_{0}}+\frac{R^{\prime}\left(a, x_{0}\right)}{x-x_{0}} .
$$


As $\left|x^{\prime}-x_{0}\right|<\delta,|x-a| \leqq\left|x-x_{0}\right|$ and $\left|x-x_{0}\right| \geqq\left|a-x_{0}\right|$,

$$
\left|\frac{f_{s}(x)-f_{s}\left(x_{0}\right)}{x-x_{0}}-f_{o+1}\left(x_{0}\right)\right|<\epsilon \quad\left(\left|x-x_{0}\right|<\delta\right),
$$

as required. (We have given here the details of A. E., Lemma 1.)

We must prove still that each $f_{0}(x)$ is continuous at each point $x_{0}$ of $A$; it is of course true in $E-A$. As $f_{s}(x)$ is continuous in $A$, it is sufficient to prove that for every $\epsilon>0$ there is a $\delta>0$ such that if $(a, b)$ is any interval of $E-A$ lying within $\delta$ of $x_{0}$, then

$$
\left|f_{8}(x)-f_{8}(a)\right|<\epsilon \quad(a \leqq x \leqq b) .
$$

Take $\epsilon^{\prime}<\epsilon /\left[2(m+1)^{2} K\right]$, where $K$ is a number to be determined later. Let $M$ be the maximum of $\left|f_{i}(x)\right|$ in $A\left(\left|x-x_{0}\right| \leqq 1, i=0, \cdots, m\right)$. Take $\delta<\epsilon /(2 m M)$ and $<1$ so small that (3.2) holds with $\epsilon$ replaced by $\epsilon^{\prime}$ for any $x$, $x^{\prime}$ within $\delta$ of $x_{0}$. Now take any interval $(a, b)$ of $E-A$ lying within $\delta$ of $x_{0}$. In $(a, b), f(x)$ equals

$$
P(x)=\sum_{i=0}^{m} \frac{f_{i}(a)}{i !}(x-a)^{i}+\sum_{i=m+1}^{2 m+1} \frac{\gamma_{i}}{i !}(x-a)^{i},
$$

where the $\gamma_{i}$ are determined by the relations

$$
\frac{d^{s}}{d x^{s}} P(b)=\sum_{i=s}^{m} \frac{f_{i}(a)}{(i-s) !}(b-a)^{i-s}+\sum_{i=m+1}^{2 m+1} \frac{\gamma_{i}}{(i-s) !}(b-a)^{i-s}=f_{s}(b) ;
$$

hence

$$
\sum_{i=m+1}^{2 m+1} \frac{\gamma_{i}}{(i-s) !}(b-a)^{i-s}=f_{s}(b)-\sum_{i=s}^{m} \frac{f_{i}(a)}{(i-s) !}(b-a)^{i-s}=R_{8}(b, a) .
$$

Solving for the $\boldsymbol{\gamma}_{i}$, we find

$$
\gamma_{i}=\sum_{j=0}^{m} K_{i j} \frac{R_{j}(b, a)}{(b-a)^{i-j}},
$$

where the $K_{i j}$ depend on $m$ alone. Set $K=\max \left|K_{i j}\right|$; then

$$
\left|\gamma_{i}\right| \leqq \sum_{j=0}^{m} \frac{K}{|b-a|^{i-m}}\left|\frac{R_{j}(b, a)}{(b-a)^{m-j}}\right|<\frac{(m+1) K}{|b-a|^{i-m}} \epsilon^{\prime} .
$$

Now if $x$ is any point in $(a, b)$, then $|x-a| \leqq|b-a|$, and 


$$
\begin{aligned}
\left|f_{\bullet}(x)-f_{s}(a)\right| & =\left|\frac{d^{\bullet}}{d x^{s}} P(x)-f_{s}(a)\right| \\
& =\left|\sum_{i=s+1}^{m} \frac{f_{i}(a)}{(i-s) !}(x-a)^{i-s}+\sum_{i=m+1}^{2 m+1} \frac{\gamma_{i}}{(i-s) !}(x-a)^{i-s}\right| \\
& <m M|x-a|+(m+1) K \epsilon^{\prime} \sum_{i=m+1}^{2 m+1} \frac{|x-a|^{i-s}}{|b-a|^{i-m}} \\
& <m M \delta+(m+1)^{2} K|b-a|^{m-s^{\prime}} \epsilon^{\prime}<\epsilon,
\end{aligned}
$$

as required.

\section{TheOREM I, $A$ PERfect}

5. A succession of lemmas culminates in Lemma 7, which is the sufficiency part of Theorem I for perfect sets.

Lemma 1. Let $A$ be a closed set, and let $\Delta^{\circ} f(x)$ converge on $A$. Then we can define $f_{*}(x)$ on the set of limit points $A^{*}$ of $A$ so that the following is true. Given $x$ in $A^{*}$ and $\epsilon>0$, we can choose $a \delta>0$ so that if $x_{0}, \cdots, x_{*}$ is any set of distinct points of $A$ lying within $\delta$ of $x$, then $\left|\Delta_{0} \ldots,-f_{\diamond}(x)\right|<\epsilon$.

The proof is simple.

Lemma 2. If $\Delta^{\circ} f_{0}(x)$ converges in the perfect set $A$, then for each point $x$ of $A$ and every $\epsilon>0$ there is $a \delta>0$ such that

$$
\left|\Delta_{0} \ldots t-1, t \cdots,-1-\Delta_{0} \cdots(t-1)^{\prime}, t \cdots,-1\right|<\epsilon
$$

$(0 \leqq t \leqq s)$ whenever all the points concerned lie within $\delta$ of $x$.

This is trivial if $t=0$. We assume it holds for numbers $0, \cdots, t-1$, and shall prove it for $t$. Given a point $x_{8}$ distinct from all former points, the equations

$$
\begin{aligned}
& \Delta_{0, \ldots l-1, \imath \cdots,-1, \ell}=\frac{s}{u_{0,}}\left(\Delta_{1} \ldots t-1, \imath \ldots,-\Delta_{0} \ldots t-1, \imath \ldots,-1\right),
\end{aligned}
$$

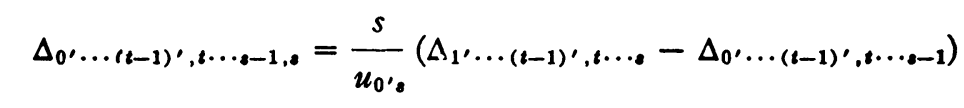

give

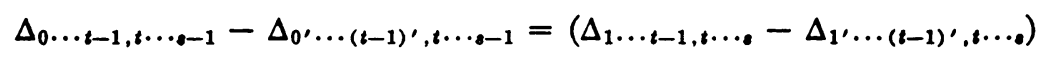

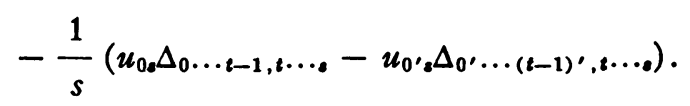

As $\Delta_{0} \ldots$ converges, we can take $M>0$ and $\delta^{\prime}<\epsilon /(4 M)$ so that $\left|\Delta_{0} \ldots.\right|<M$ whenever $x_{0}, \cdots, x_{s}$ are within $\delta^{\prime}$ of $x$. By induction, we can take $\delta<\delta^{\prime}$ so small that the first term on the right in (5.2) is in absolute value $<\epsilon / 2$ whenever all points concerned are within $\delta$ of $x$. Now given the points $x_{0}, \cdots, x_{\bullet-1}$, 
$x_{0^{\prime}}, \cdots, x_{(t-1)^{\prime}}$ within $\delta$ of $x$, let $x_{\varepsilon}$ be another such point; then (5.2) gives (5.1).

The lemma with $t=s$ shows that $\Delta^{s-1} f_{0}(x)$ converges in $A$.

LEMMA 3. If $\Delta^{m} f_{0}(x)$ converges in the perfect set $A$, then there are continuous functions $f_{1}(x), \cdots, f_{m}(x)$ in $A$ such that $\Delta^{\circ} f_{0}(x) \rightarrow f_{s}(x)$ in $A(s=1, \cdots, m)$.

We prove this successively for $s=m, m-1, \cdots, 1$ with the help of Lemmas 1 and 2.

6. We proceed to the following lemma.

Lемма 4. If $\Delta^{p} g(x) \rightarrow g_{p}(x)$ and $\Delta^{1} g(x) \rightarrow g_{1}(x)$ in the perfect set $A$, then $\Delta^{p-1} g_{1}(x) \rightarrow g_{p}(x)$ in $A$.

Set $q=p-1$. If we apply the relation $\Delta^{1} \phi\left(x_{i}\right) \rightarrow d \phi\left(x_{i}\right) / d x_{i}$ to $\alpha_{0}^{i} \ldots i \ldots q$ as a differentiable function of $x_{i}$, we find

$$
\frac{\alpha_{0}^{i^{\prime} \ldots i^{\prime} \cdots q}-\alpha_{0}^{i} \ldots q}{u_{i i^{\prime}}}=-\alpha_{0, \ldots q}^{i} \sum_{j \ngtr i} \frac{1}{u_{j i}}+\epsilon\left(x_{i^{\prime}}\right),
$$

where $\epsilon\left(x_{i^{\prime}}\right) \rightarrow 0$ as $x_{i^{\prime}} \rightarrow x_{i}$. Hence

$$
\begin{aligned}
\alpha_{0 \cdots q}^{i} \sum_{i} \frac{1}{u_{j^{\prime} i}} & =-\frac{\alpha_{0 \cdots i^{\prime} \cdots q}^{i^{\prime}}}{u_{i i^{\prime}}}+\alpha_{0 \cdots q}^{i} \sum_{j \neq i}\left(\frac{1}{u_{j^{\prime} i}}-\frac{1}{u_{j^{\prime}}}\right)+\epsilon\left(x_{i^{\prime}}\right) \\
& =-\frac{\alpha_{0}^{i^{\prime} \cdots i^{\prime} \cdots q}}{u_{i^{\prime}}}+\zeta_{i}\left(x_{0^{\prime}}, \cdots, x_{q^{\prime}}\right),
\end{aligned}
$$

where $\zeta_{i}\left(x_{0^{\prime}}, \cdots, x_{q^{\prime}}\right) \rightarrow 0$ as $x_{j^{\prime}} \rightarrow x_{j}(j=0, \cdots, q)$. Consider the $2 q$ points $x_{0}, x_{0^{\prime}}, \cdots, x_{q}, x_{q^{\prime}}$. We have

$$
\begin{aligned}
& \Delta_{0 \ldots i j^{\prime} \cdots q} g=p !\left[\sum_{i=0}^{q} \frac{\alpha_{0 \cdots q}^{i}}{u_{j^{\prime} i}} g\left(x_{i}\right)+\frac{\alpha_{0 \cdots j^{\prime} \cdots q}^{j^{\prime}}}{u_{j^{\prime}}} g\left(x_{j^{\prime}}\right)\right], \\
& \frac{1}{p} \sum_{j=0}^{q} \Delta_{0 \ldots i j^{\prime} \ldots q} g=(p-1) !\left[\sum_{i=0}^{q} \alpha_{0 \cdots q}^{i} g\left(x_{i}\right) \sum_{j=0}^{q} \frac{1}{u_{j^{\prime} i}}+\sum_{i=0}^{q} \frac{\alpha_{0 \cdots i^{\prime} \cdots q}^{i^{\prime}}}{u_{i i^{\prime}}} g\left(x_{i^{\prime}}\right)\right] \\
& =q ! \sum_{i=0}^{q}\left[\alpha_{0, \ldots i^{\prime} \cdots q}^{i^{\prime}} \frac{g\left(x_{i^{\prime}}\right)-g\left(x_{i}\right)}{u_{i i^{\prime}}}+g\left(x_{i}\right) \zeta_{i}\left(x_{0^{\prime}}, \cdots, x_{q^{\prime}}\right)\right] \text {. }
\end{aligned}
$$

As $\Delta^{1} g(x) \rightarrow g_{1}(x)$, this gives, letting $x_{j^{\prime}} \rightarrow x_{j}(j=0, \cdots, q)$,

$$
\frac{1}{p} \lim \sum_{j=0}^{q} \Delta_{0} \ldots j j^{\prime} \cdots q g=q ! \sum_{i=0}^{q} \alpha_{0}^{i} \cdots_{q} g_{1}\left(x_{i}\right)=\Delta_{0} \ldots q g_{1} .
$$


Now given a point $x$ of $A$ and an $\epsilon>0$, take $\delta>0$ so that if $x_{0}, \cdots, x_{i}$, $x_{i^{\prime}}, \cdots, x_{q}$ are within $\delta$ of $x$, then

$$
\left|\Delta_{0} \ldots i j^{\prime} \cdots q g-g_{p}(x)\right|<\epsilon .
$$

Then if $x_{0}, \cdots, x_{q}$ are within $\delta$ of $x$, we find by adding points $x_{0^{\prime}}, \cdots, x_{q^{\prime}}$ within $\delta$ of $x$ and letting $x_{i^{\prime}} \rightarrow x_{i}(i=0, \cdots, q)$ that

as required.

$$
\left|\Delta_{0} \ldots q g_{1}-g_{p}(x)\right|<\epsilon,
$$

LEMMA 5. If $\Delta^{m} f_{0}(x)$ converges in the perfect set $A$, then there are continuous functions $f_{1}(x), \cdots, f_{m}(x)$ such that $\Delta_{p} f_{q}(x) \rightarrow f_{p+q}(x)$ in $A$.

This follows from Lemmas 3 and 4.

7. We now present the two final lemmas needed for the proof of Theorem I when $A$ is perfect.

Lemma 6. Let $g(x)=g_{0}(x), \cdots, g_{\circ}(x)$ be defined in the perfect set $A$, and suppose $\Delta \cdot g(x) \rightarrow g_{\bullet}(x)$. If $g(x)$ can be expanded in a Taylor's formula to the $(s-1)$ th order in terms of $g_{0}(x), \cdots, g_{\bullet-1}(x)$, then it can be expanded in a Taylor's formula to the sth order locally uniformly in terms of $g_{0}(x), \cdots, g_{s}(x)$.

Given a point $x$ of $A$ and an $\epsilon>0$, take $\delta>0$ so that

$$
\left|\Delta_{0} \ldots s-g_{s}\left(x_{0}\right)\right|<\frac{s !}{2 s} \frac{\epsilon}{3}
$$

whenever $x_{0}, \cdots, x_{s}$ are within $\delta$ of $x$ (recall that $g_{s}(x)$ is continuous, by $\S 2$ ). Take any two points $x_{0}$ and $x_{8}$ of $A$ within $\delta$ of $x$; we must show that $\left|R^{(o)}\left(x_{s}, x_{0}\right)\right| / r_{0 s}^{s}<\epsilon$.

Take $\delta^{\prime}$ so small that if $\left|x^{\prime}-x_{0}\right|<\delta^{\prime}$, then

$$
\left|\frac{R^{(s-1)}\left(x^{\prime}, x_{0}\right)}{\left(x^{\prime}-x_{0}\right)^{s-1}}\right|<\frac{r_{0 s}}{2^{2 s s}} \frac{\epsilon}{3},
$$

where $R^{(\bullet-1)}\left(x^{\prime}, x_{0}\right)=g\left(x^{\prime}\right)-\sum_{j=0}^{s-1} g_{j}\left(x_{0}\right)\left(x^{\prime}-x_{0}\right)^{i} / j !$. Take $M>\left|g_{\star}\left(x_{0}\right)\right|$. Take a point $x_{s-1}$ in $A$ within $\delta^{\prime}$ of $x_{0}$ and so close to $x_{0}$ that

$$
\frac{r_{0,8-1}}{r_{0,}}<\frac{s !}{2 \cdot M} \frac{\epsilon}{3} \text { and }<\frac{1}{2}
$$

and (if $s>2$ ) take in succession points $x_{8-2}, \cdots, x_{1}$ in $A$ so that

$$
r_{0, t-1}<\frac{1}{2} r_{0 t} \quad(t=2 . \cdots, s-1) ;
$$

let these points lie within $\delta$ of $x$. Then if $i<s$, 


$$
\left|\alpha_{0 \ldots s}^{i} R^{(s-1)}\left(x_{i}, x_{0}\right)\right|<\frac{1}{r_{s i}} \frac{r_{0 i}^{s-1}}{r_{0 i} \cdots r_{i-1, i} r_{i+1, i} \cdots r_{s-1, i}} \frac{r_{0 s}}{2^{2 s s}} \frac{\epsilon}{3}<\frac{1}{2^{s} s} \frac{\epsilon}{3} .
$$

Now

$$
\begin{aligned}
\frac{1}{s !} \Delta_{0} \ldots s & =\alpha^{8} g\left(x_{8}\right)+\sum_{i=0}^{s-1} \alpha^{i}\left[\sum_{j=0}^{s-1} \frac{g_{j}\left(x_{0}\right)}{j !} u_{0 i}^{j}+R^{(8-1)}\left(x_{i}, x_{0}\right)\right] \\
& =\alpha^{8} g\left(x_{s}\right)-\alpha^{8} \sum_{j=0}^{s-1} \frac{g_{j}\left(x_{0}\right)}{j !} u_{0 s}^{j}+\sum_{i=0}^{s-1} \alpha^{i} R^{(8-1)}\left(x_{i}, x_{0}\right),
\end{aligned}
$$

on account of (2.5). Therefore

$$
\begin{aligned}
R^{(s)}\left(x_{s}, x_{0}\right) & =g\left(x_{s}\right)-\sum_{j=0}^{s} \frac{g_{j}\left(x_{0}\right)}{j !} u_{0 s}^{j} \\
& =\frac{\Delta_{0} \ldots s}{s ! \alpha^{s}}-\frac{g_{s}\left(x_{0}\right) u_{0 s}^{e}}{s !}-\sum_{i=0}^{s-1} \frac{\alpha^{i}}{\alpha^{8}} R^{(s-1)}\left(x_{i}, x_{0}\right),
\end{aligned}
$$

and as $r_{i s} / r_{0 s} \leqq\left(r_{0 s}+r_{0 i}\right) / r_{0 s}=1+r_{0 i} / r_{0 s}$,

$$
\begin{aligned}
\frac{\left|R^{(s)}\left(x_{s}, x_{0}\right)\right|}{r_{0 s}^{s}} \leqq & \frac{r_{0 s} \cdots r_{s-1, s}}{s ! r_{0 s}^{s}}\left|\Delta_{0, \ldots s}-g_{s}\left(x_{0}\right)\right|+\frac{\left|g_{s}\left(x_{0}\right)\right|}{s !}\left|\frac{r_{0 s} \cdots r_{s-1, s}}{r_{0 s}}-1\right| \\
& +\sum_{i=0}^{s-1} \frac{r_{0 s} \cdots r_{s-1, s}}{r_{0 s}}\left|\alpha^{i} R^{(o-1)}\left(x_{i}, x_{0}\right)\right| \\
<\frac{2 s}{s !} \frac{s !}{2 s} \frac{\epsilon}{3} & +\frac{M}{s !}\left[\left(1+\frac{r_{00}}{r_{0 s}}\right) \cdots\left(1+\frac{r_{0, s-1}}{r_{0 s}}\right)-1\right]+s \cdot 2^{s} \frac{1}{2 s s} \frac{\epsilon}{3}<\epsilon,
\end{aligned}
$$

as required.

LEMMA 7. If $\Delta^{m} f(x)$ converges in the perfect set $A$, then $f_{0}(x)=f(x)$, $f_{1}(x), \cdots, f_{m}(x)$ can be defined in $A$ so that $f(x)$ is of class $C^{m}$ in $A$ in terms of the $f_{0}(x)(s=0, \cdots, m)$.

We define $f_{1}(x), \cdots, f_{m}(x)$ by means of Lemma 5. Taylor's formula for each $f_{s}(x)$ holds to the 0th order, as $f_{s}(x)$ is continuous (see $\left.\$ 2\right)$. We prove in succession that it holds to the $k$ th order for $k=1, \cdots, m-s$. This completes the proof of the lemma, and therefore of Theorem $I$ for the case that $A$ is perfect.

\section{$P$-SETS AND $Q$-SETS}

8. We shall prove a lemma which will be needed in the next part. Let $A^{\prime}=a_{1}, a_{2}, \cdots$ be a set of isolated points, at least $m+1$ in number. With each point $a_{i}$ we shall associate $m$ other points $a_{i_{1}}, \cdots, a_{i_{m}}$; these $m$ points 
together with $a_{i}$ we say form the $Q$-set $Q\left(a_{i}\right)$. Take a $Q$-set $Q_{i}$, and let $a_{j_{1}}, \cdots, a_{j_{\text {}}}$ be all those points such that $Q\left(a_{i_{t}}\right)=Q_{i}$; these points form the $P$-set $P_{i}$ corresponding to $Q_{i}$. Each point of $P_{i}$ is in $Q_{i}$. Each point $a_{i}$ lies in just one $P$-set $P\left(a_{i}\right)$, as $a_{i}$ is associated with just one $Q$-set $Q\left(a_{i}\right)$; however, $a_{i}$ may lie in several $Q$-sets. Let $\delta\left(Q_{i}\right)$ be the greatest distance between pairs of points of $Q_{i}$.

LEMMA 8. The P-sets and $Q$-sets may be so chosen that for any two points $a_{i}$ and $a_{j}$,

$$
\text { if } \frac{\delta\left(Q\left(a_{i}\right)\right)+\delta\left(Q\left(a_{j}\right)\right)}{\left|a_{i}-a_{i}\right|}>2 m, \text { then } P\left(a_{i}\right)=P\left(a_{i}\right)
$$

We first associate sets of points with certain of the limit points of the points $a_{1}, a_{2}, \cdots$ as follows. Let $c_{i}$ be a point such that there is a sequence of points of $A^{\prime}$ approaching it from one side, say the left, while there is a nearest point of $A^{\prime}$ to $c_{i}$ on the other side of $c_{i}$. Let $\mu$ equal $m+1$, or the number of points $a_{j}$ between $c_{i}$ and the next limit point $c_{k}$ to the right of $c_{i}$ if that number is smaller, and let $a_{j_{1}}, \cdots, a_{i_{\mu}}$ be the points nearest $c_{i}$ on the right (counting from left to right). Let $\tau$ be the smallest of the numbers $\left|a_{j}-a_{i_{\text {s }}}\right|$ $(s, t=1, \cdots, \mu)$ which are $>\left|a_{i_{1}}-c_{i}\right|$, if there are such. Let $a_{1}\left(c_{i}\right)$ be a point of $A^{\prime}$ to the left of $c_{i}$ such that

$$
\left|c_{i}-a_{1}\left(c_{i}\right)\right|<\left|a_{i_{1}}-c_{i}\right|, \text { and }\left|a_{i_{1}}-a_{1}\left(c_{i}\right)\right|<\tau
$$

if $\tau$ is defined. Let $a_{2}\left(c_{i}\right), \cdots, a_{m}\left(c_{i}\right)$ be points of $A^{\prime}$ lying between $c_{i}$ and $a_{1}\left(c_{i}\right)$.

We now define the $Q$-sets. Given a point $a_{i}$, we associate another point with it as follows. Suppose, Case I, there is a point $a_{i}$ whose distance from $a_{i}$ is less than or equal to the distance from any other $a_{k}$ to $a_{i}$; then we associate $a_{j}$ with $a_{i}$, or that one of the pair $a_{j}, a_{k}$ which lies to the left of $a_{i}$, if their distances from $a_{i}$ are the same. Suppose, Case II, there is no such point. Then there is a limit point $c_{i}$ nearer $a_{i}$ than any point $a_{j}$. If there are two such points, we consider that one $c_{i}$ on the left. The point we associate with $a_{i}$ is then $a_{1}\left(c_{i}\right)$.

Suppose now we have associated a number of points with $a_{i}$, forming the set of points $S$. We associate the next point in a fashion much the same as above. If Case II has not occurred in associating the other points of $S$ with $a_{i}$, we again have two cases to consider. Case I, there is a nearest point $a_{i}$ to the set $S$; we then associate this point with $S$ (or the point $a_{k}$, as above). Case II, there is none; then take the point $c_{i}$ as above, and associate $a_{1}\left(c_{i}\right)$ with $S$. At any time we employ Case II, we immediately associate also the 
points $a_{2}\left(c_{i}\right), a_{3}\left(c_{i}\right), \ldots$ with $S$, till we have the required $m+1$ points $Q\left(a_{i}\right)$.

Note that the point we associate with $S$ does not depend on which point $a_{i}$ of $S$ we started with. Also if Case I has occurred each time in forming the subset $S$ of $Q\left(a_{i}\right)$, then there is no point $a_{k}$ not in $S$ which lies between two points of $S$.

9. To prove that (8.1) holds take any two points $a_{i}$ and $a_{j}$; set $r_{i j}=\left|a_{j}-a_{i}\right|$.

(1) Suppose there are at most a finite number of points of $A^{\prime}$ between $a_{i}$ and $a_{j}$. If $\delta\left(Q\left(a_{i}\right)\right)+\delta\left(Q\left(a_{j}\right)\right)>2 m r_{i j}$, then either $\delta\left(Q\left(a_{i}\right)\right)>m r_{i j}$ or $\delta\left(Q\left(a_{j}\right)\right)$ $>m r_{i j}$, say the former. Then there is a first time when, on adding a point $a_{k}$ to a set $S$ in forming $Q\left(a_{i}\right)$, the distance from $a_{k}$ to $S$ is $>r_{i j}$.

(a) In forming $S$ from $a_{i}$, Case I has occurred each time. For if Case II had occurred, say in adding the point $a_{1}\left(c_{i}\right)$ to the subset $S_{1}$ of $S$, then $a_{k}$ would be some $a_{s}\left(c_{i}\right)$; but the distance from $a_{k}$ to $S$ is then at most the distance from $a_{s}\left(c_{i}\right)$ to $a_{1}\left(c_{i}\right)$ which is less than the distance from $a_{1}\left(c_{i}\right)$ to $S_{1}$ which is by hypothesis $\leqq r_{i j}$.

(b) There is no point $a_{s}$ whose distance from $S$ is $\leqq r_{i j}$. For suppose there were; then Case II must occur in adding $a_{k}=a_{1}\left(c_{i}\right)$ to $S$, and $c_{i}$ is nearer $S$ than any point $a_{r}$. (If Case I occurred, $a_{s}$ or a nearer point, not $a_{k}$, would be added to $S$.) Say $c_{i}$ lies to the left of $S$. Let $a_{p}$ and $a_{q}$ be the left and righthand end points of $S$ respectively. As there is a point $a_{s}$ distant $\leqq r_{i j}$ from $S$, $\left|a_{p}-c_{i}\right|<r_{i j}$. Suppose $a_{j}$ is not in $S$. As there are no limit points between $a_{i}$ and $a_{j}, a_{j}$ lies to the right of $S$, and hence there is a first point $a_{r}$ to the right of $S$. Then as $a_{i}$ is in $S,\left|a_{r}-a_{q}\right| \leqq r_{i j}$. But as $a_{q}$ and $a_{r}$ are among the first $m+1$ (or $\mu$ ) points to the right of $c_{i}$, and $\left|a_{p}-c_{i}\right|<\left|a_{r}-a_{q}\right|$, (8.2) gives $\left|a_{p}-a_{k}\right|<\left|a_{r}-a_{q}\right| \leqq r_{i j}$, a contradiction; therefore $a_{j}$ is in $S$. As $a_{j}$ is in $S$ and $\left|a_{p}-c_{i}\right|<r_{i j},(8.2)$ gives $\left|a_{p}-a_{k}\right|<r_{i j}$, again a contradiction.

(c) $S$ contains $a_{j}$. For otherwise (b) would be contradicted.

(d) In forming $Q\left(a_{j}\right)$, the points of $S$ are chosen first. For suppose not. Then after perhaps adding some points of $S$ to $a_{j}$, forming the set $S^{\prime}$, we choose a point $a_{l}$ not in $S$. By (b), the distance from $a_{l}$ to $S$ is $>r_{i j}$. As there is a point in $S$ whose distance from $S^{\prime}$ is at most $r_{i j}, a_{l}$ must have been chosen under Case II; then the distance from some point $c_{j}$ to $S^{\prime}$ is $\left\langle r_{i j}\right.$. But then as $c_{j}$ is a limit point of points $a_{s}$, there is a point $a_{s}$ whose distance from $S$ is $<r_{i j}$, a contradiction.

Now in forming both $Q\left(a_{i}\right)$ and $Q\left(a_{i}\right)$, the points of $S$ are chosen first. As the remaining points chosen depend only on $S, Q\left(a_{i}\right)$ and $Q\left(a_{j}\right)$ must coincide; hence $a_{i}$ and $a_{j}$ lie in the same $P$-set.

(2) Suppose there is a limit point of isolated points $b$ between $a_{i}$ and $a_{j}$. In forming $Q\left(a_{i}\right)$, the set $S$ at any step is at a distance $\leqq\left|b-a_{i}\right|$ from $b$; hence in adding the next point $a_{k}$ to $S$, its distance from $S$ is $\leqq\left|b-a_{i}\right|$ if Case I 
occurs, and is $<2\left|b-a_{i}\right|$ if Case II occurs, by (8.2). Therefore $\delta\left(Q\left(a_{i}\right)\right)$ $<2 m\left|b-a_{i}\right|$. Similarly $\delta\left(Q\left(a_{j}\right)\right)<2 m\left|b-a_{j}\right|$. Adding,

$$
\delta\left(Q\left(a_{i}\right)\right)+\delta\left(Q\left(a_{i}\right)\right)<2 m\left(\left|b-a_{i}\right|+\left|b-a_{i}\right|\right)=2 m r_{i j},
$$

completing the proof.

Remark. Given a point $a_{i}$, if there exist $m$ points $a_{i_{1}}, \cdots, a_{i_{m}}$ such that the $m$ intervals between $a_{i}, a_{i_{1}}, \cdots, a_{i_{m}}$ are all $\leqq \rho$, or if there exists a point $a$ not in $Q\left(a_{i}\right)$ within $\rho$ of $a_{i}$, then $\delta\left(Q\left(a_{i}\right)\right)<2 m \rho$. This follows from the proof in (2).

\section{Theorem I, $A$ CLOSED}

Each isolated point of $A$ is enclosed in an interval; this gives a perfect set $B$. The definition of $f(x)$ is extended over $B$. With the help of Lemma 8 it is shown that $\Delta^{m} f(x)$ now converges over $B$. By Lemma $7, f(x)$ is of class $C^{m}$ in $B$; hence the same is true in $A$.

10. The sets $A^{\prime}$ and $B$. Let $A_{1}$ be the set of isolated points of the closed set $A$, let $A_{2}$ be the set of limit points of isolated points, and let $A_{3}$ be the remaining points of $A$. Let $A^{\prime}$ consist of $A_{1}$, together with certain other points as follows. $A_{1}+A_{2}$ being closed, let $I$ be any open interval of $E-\left(A_{1}+A_{2}\right)$ containing points of $A_{3}$. If an end point $a_{i}$ of $I$ is in $A_{1}$, then there is, in $I$, a nearest point $a_{1}\left(a_{i}\right)$ of $A_{3}$ to $a_{i}$. We associate this point with $a_{i}$, and also points $a_{2}\left(a_{i}\right), \cdots, a_{m}\left(a_{i}\right)$ of $A_{3}$ in $I$, chosen so that

$$
\left|a_{s}\left(a_{i}\right)-a_{1}\left(a_{i}\right)\right|<\left|a_{1}\left(a_{i}\right)-a_{i}\right| \quad(s=2, \cdots, m) .
$$

$A^{\prime}$ is a set of isolated points; we may name them $a_{1}, a_{2}, \cdots . A^{\prime}$ is contained in $A_{1}+A_{3}$.

For each point $a_{i}$ of $A_{1}$, let $d\left(a_{i}\right)$ be its distance from the rest of $A$, and let $B_{i}$ be a closed interval of length $d\left(a_{i}\right) / 2$, with $a_{i}$ as center. Let the perfect set $B$ be $A$ plus all of these intervals. Arrange the points of $A^{\prime}$ into $P$-sets and $Q$-sets so as to obey Lemma 8 . For each $P$-set $P_{i}$, let the corresponding $P^{\prime}$-set $P_{i}^{\prime}$ contain the points of $P_{i}$, together with the points of any intervals $B_{i}$ there may be which enclose points of $P_{i}$.

Given any set $S$ of $m+1$ points in $B$, we shall define its complexity $\sigma(S)$ as follows. If all the points of $S$ are in $A$, set $\sigma(S)=0$. If $S$ contains $p>0$ points in $B-A$, and all these points lie in a single $P^{\prime}$-set $P_{i}^{\prime}$, let $q$ be the number of remaining points of $S$ which do not lie in the corresponding $Q$-set $Q_{i}$, and set $\sigma(S)=p q$. The complexity of $S$ is in this case certainly $\leqq m^{2}$. If $S$ contains $p$ points in $B-A$, and these points do not all lie in the same $P^{\prime}$-set, set $\sigma(S)=m^{2}+p-1$. The complexity of any set $S$ is $\leqq m^{2}+m$.

11. The following lemma together with Lemma 7 gives Theorem I. 
Lemma 9. Let $f(x)$ be defined in the closed set $A$ so that $\Delta^{m} f(x)$ converges in $A$. Then $A$ can be enclosed in a perfect set $B$, the definition of $f(x)$ can be extended over $B$, and $f_{m}(x)$ can be defined in $B$, so that $\Delta^{m} f(x) \rightarrow f_{m}(x)$ in $B$.

Define the sets $A^{\prime}, B$ etc. as above. We may assume there are at least $m+1$ points in $A^{\prime}$. Define $f_{m}(x)$ at each point of $A_{2}+A_{3}$ as in Lemma 1. Take a fixed interval $B_{i}$ with center $a_{i}$; we define $f(x)$ and $f_{m}(x)$ over $B_{i}$ as follows. Let $Q_{i}=Q\left(a_{i}\right)$ be the corresponding $Q$-set. Let

$$
R_{i}(x)=\gamma_{0}+\cdots+\gamma_{m} x^{m}
$$

be the polynomial of degree at most $m$ such that $R_{i}(x)=f(x)$ at each point of $Q_{i}$; then $\Delta\left(Q_{i}\right)=m ! \gamma_{m}$, by (2.7). Set

$$
f(x)=R_{i}(x), \quad f_{m}(x)=m ! \gamma_{m} \text { in } B_{i} .
$$

The same polynomial $R_{i}(x)$ is used in defining $f(x)$ and $f_{m}(x)$ over each interval of the $P^{\prime}$-set $P_{i}^{\prime}$ corresponding to $Q_{i}$; hence if $S$ is any set of $m+1$ points such that all of its points in $B-A$ lie in $P_{i}^{\prime}$, and all remaining points lie in $Q_{i}$, then $f(x)=R_{i}(x)$ at each point of $S$, and therefore, by $(2.7), \Delta(S)$ $=m ! \gamma_{m}=\Delta\left(Q_{i}\right)$.

Each point $x$ of $A_{3}$ is at a positive distance from $B-A$; by the definition of $f_{m}(x), \Delta^{m} f(x) \rightarrow f_{m}(x)$ at such points. Each point $x$ of $B-\left(A_{2}+A_{3}\right)$ is in an interval $B_{i}$; hence near $x, f(x)$ is a polynomial, and $\Delta^{m} f(x) \rightarrow f_{m}(x)$ there also. It remains to show that for each point $x$ of $A_{2}$ and every $\epsilon>0$ there is a $\delta>0$ such that if $S$ is any set of $m+1$ points of $B$ within $\delta$ of $x$, then

$$
\left|\Delta(S)-f_{m}(x)\right|<\epsilon .
$$

By Lemma 1, we can take $\delta^{\prime}>0$ so that

$$
\left|\Delta\left(S_{0}\right)-f_{m}(x)\right|<\frac{\epsilon}{(8 m+8)^{m^{2}+m}}
$$

for any set $S_{0}$ of $m+1$ points of $A$ lying within $\delta^{\prime}$ of $x$. Set $\delta=\delta^{\prime} /(4 m+2)$. We shall prove the following:

(A) If $S$ is any set in $B$, of complexity $\sigma(S)=\sigma$, composed of sets of points $S_{1}$ in $B-A$ and $S_{2}$ in $A$, and if $S_{1}$ lies within $\delta$ of $x$ and $S_{2}$ lies within $\delta^{\prime}$ of $x$, then

$$
\left|\Delta(S)-f_{m}(x)\right|<\epsilon_{\sigma}=\frac{\epsilon}{(8 m+8)^{m^{2}+m-\sigma}} .
$$

As $\sigma \leqq m^{2}+m,(11.3)$ follows.

12. We note first that if $b_{i}$ is in some interval of the $P^{\prime}$-set $P_{i}^{\prime}$, and $b_{i}$ lies within $\delta$ of $x$, then $Q_{i}$ lies within $\delta^{\prime}$ of $x$. Say $a_{i}$ is the center of $B_{i}$; then $a_{i}$ lies 
within $2 \delta$ of $x$, a limit point of points of $A^{\prime}$. Hence $\delta\left(Q\left(a_{i}\right)\right)<4 m \delta$, by the remark at the end of $\S 9$, and $Q_{i}=Q\left(a_{i}\right)$ lies within $(4 m+2) \delta=\delta^{\prime}$ of $x$.

We shall prove (A) first for $\sigma=0$, then for $\sigma>0$, using induction. Suppose $\sigma=0$. If $S$ is in $A$, the fact follows from (11.4). If $S$ contains points of $B-A$, then all these points lie in a single $P^{\prime}$-set $P_{i}^{\prime}$, and the rest of $S$ lies in the corresponding $Q$-set $Q_{i}$; hence $\Delta(S)=\Delta\left(Q_{i}\right) . Q_{i}$ lies within $\delta^{\prime}$ of $x$; hence (11.4) holds with $S_{0}$ replaced by $Q_{i}$ or by $S$, and therefore (11.5) holds.

Now suppose (11.5) is proved for all sets $S^{\prime}$ with $\sigma\left(S^{\prime}\right)<\sigma$; we shall prove it for any set $S$ with $\sigma(S)=\sigma$. Suppose first $\sigma>m^{2}$; then the points of $S$ in $B-A$ lie in at least two $P^{\prime}$-sets. Let $P_{i}^{\prime}$ and $P_{i}^{\prime}$ be two of these sets, let $b_{i}$ and $b_{j}$ be points of $S$ (in $B-A$ ) in $P_{i}^{\prime}$ and $P_{j}^{\prime}$ respectively, and let $a_{i}$ and $a_{j}$ be the centers of the corresponding intervals. Let $a_{k}$ be a point of $Q\left(a_{i}\right)$ not lying in $S$. If $S^{\prime}=S-b_{i}-b_{j}$, then, by (2.4),

$$
\Delta(S)=\Delta\left(b_{i}, b_{j}, S^{\prime}\right)=\frac{a_{k}-b_{i}}{b_{j}-b_{i}} \Delta\left(b_{i}, a_{k}, S^{\prime}\right)+\frac{b_{j}-a_{k}}{b_{i}-b_{i}} \Delta\left(a_{k}, b_{j}, S^{\prime}\right) .
$$

The sets $S^{\prime}+b_{i}+a_{k}$ and $S^{\prime}+b_{j}+a_{k}$ each contain fewer points of $B-A$ than $S$; hence their complexities are each $<\sigma$. Also $Q\left(a_{i}\right)$ and therefore $a_{k}$ lie within $\delta^{\prime}$ of $x$. Therefore, by induction,

$$
\left|\Delta\left(b_{i}, a_{k}, S^{\prime}\right)-f_{m}(x)\right|<\epsilon_{\sigma-1}, \quad\left|\Delta\left(a_{k}, b_{j}, S^{\prime}\right)-f_{m}(x)\right|<\epsilon_{\sigma-1} .
$$

As $a_{i}$ and $a_{j}$ lie in distinct $P$-sets, $\delta\left(Q\left(a_{i}\right)\right)+\delta\left(Q\left(a_{i}\right)\right) \leqq 2 m r_{i j}$, by (8.1). As $\left|b_{i}-a_{i}\right| \leqq r_{i j} / 4$ and $\left|b_{j}-a_{j}\right| \leqq r_{i j} / 4,\left|b_{j}-b_{i}\right| \geqq r_{i j} / 2$. As $a_{k}$ and $a_{j}$ lie in $Q\left(a_{i}\right)$ and $Q\left(a_{j}\right)$ respectively, $\left|a_{j}-a_{k}\right| \leqq \delta\left(Q\left(a_{i}\right)\right)+\delta\left(Q\left(a_{j}\right)\right)+r_{i j} \leqq(2 m+1) r_{i j}$; hence $\left|b_{j}-a_{k}\right|<(2 m+2) r_{i j}$. Also $\left|a_{k}-b_{i}\right| \leqq \delta\left(Q\left(a_{i}\right)\right)+\left|a_{i}-b_{i}\right|<(2 m+2) r_{i j}$; hence

$$
\left|\frac{a_{k}-b_{i}}{b_{j}-b_{i}}\right|<4 m+4, \quad\left|\frac{b_{j}-a_{k}}{b_{j}-b_{i}}\right|<4 m+4 \text {. }
$$

This with (12.2) and (12.1) gives

$$
\begin{aligned}
\left|\Delta(S)-f_{m}(x)\right|< & \left|\frac{a_{k}-b_{i}}{b_{j}-b_{i}}\right|\left|\Delta\left(b_{i}, a_{k}, S^{\prime}\right)-f_{m}(x)\right| \\
& +\left|\frac{b_{j}-a_{k}}{b_{i}-b_{i}}\right|\left|\Delta\left(a_{k}, b_{j}, S^{\prime}\right)-f_{m}(x)\right| \\
& <(8 m+8) \epsilon_{\sigma-1}=\epsilon_{\sigma},
\end{aligned}
$$

as required.

Suppose now $0<\sigma \leqq m^{2}$; then the points of $S$ in $B-A$ lie in a single $P^{\prime}$-set $P_{i}^{\prime}$, and there are points of $S$ not in $P_{i}^{\prime}+Q_{i}$. Let $b_{i}$ be a point of $S$ in $B-A$, let $a$ be a point of $S$ not in $P_{i}^{\prime}+Q_{i}$, and let $a_{k}$ be a point of $Q_{i}$ which is not in $S$. If $S^{\prime}=S-b_{i}-a$, the sets $S^{\prime}+b_{i}+a_{k}$ and $S^{\prime}+a+a_{k}$ each have a smaller 
complexity than $S . a_{k}$ lies within $\delta^{\prime}$ of $x$, and hence, by induction, (12.2) holds with $b_{i}$ replaced by $a$. Let $a_{i}$ be the center of the interval $B_{i}$ containing $b_{i}$.

Suppose, (1), $a=a_{j}$ is in $A^{\prime}$. Then $\left|a_{j}-b_{i}\right|>r_{i j} / 2$. As $a_{k}$ is in $Q_{i}=Q\left(a_{i}\right)$ while $a_{j}$ is not, $\left|a_{k}-b_{i}\right|<\left|a_{k}-a_{i}\right|+r_{i j}<(2 m+1) r_{i j}$, by the remark, and $\left|a_{j}-a_{k}\right|<(2 m+1) r_{i j}$. Hence (12.3) holds with $b_{j}$ replaced by $a_{j}=a$, and (11.5) follows just as before. Suppose, (2), $a$ is in $A-\left(A^{\prime}+A_{2}\right)$. From $a$, move toward $a_{i}$ to the first point $a^{\prime}$ in $A_{1}+A_{2}$. If $a^{\prime}$ is in $A_{1}$, move back to the first point $a_{1}\left(a^{\prime}\right)$ in $A_{3}$. Then $\left|a_{1}\left(a^{\prime}\right)-a^{\prime}\right| \leqq\left|a-a_{i}\right|$ and $\left|a_{s}\left(a^{\prime}\right)-a_{1}\left(a^{\prime}\right)\right|<\mid a_{1}\left(a^{\prime}\right)$ $-a^{\prime}|\leqq| a-a_{i} \mid(s=2, \cdots, m)$, by (10.1). Hence $\delta\left(Q_{i}\right)<2 m\left|a-a_{i}\right|$, by the remark, and $\left|a_{k}-b_{i}\right|<(2 m+1)\left|a-a_{i}\right|$, and $\left|a-a_{k}\right|<(2 m+1)\left|a-a_{i}\right|$. As $\left|a-b_{i}\right|>\left|a-a_{i}\right| / 2,(12.3)$ and (11.5) follow, as before. If $a^{\prime}$ is in $A_{2}$, there are $m$ points of $A^{\prime}$ nearer $a_{i}$ than $a$, and again $\delta\left(Q_{i}\right)<2 m\left|a-a_{i}\right|$ and (11.5) follows. Suppose finally, (3), $a$ is in $A_{2}$. Again we must have $\delta\left(Q_{i}\right)<2 m\left|a-a_{i}\right|$ and (11.5) follows. This completes the proof of (A), therefore of Lemma 9, and therefore of Theorem $I$.

\section{TAYLOR'S FORMULA}

13. Conditions under which Taylor's formula is valid. Taylor's formula for $f(x)$ may hold to the $m$ th order in certain closed sets even if $f(x)$ is not of class $C^{m}$ (see \$14). We find here a difference quotient condition equivalent to the validity of Taylor's formula, at least for perfect sets.

LEMMA 10. If $f(x)=f_{0}(x)$ can be expanded in a Taylor's formula to the mth order locally uniformly in terms of $f_{0}(x), \cdots, f_{m}(x)$ in the closed set $A$, then these functions are continuous in $A$.

It is apparent from (3.1) and (3.2) with $s=0$ that $f_{0}(x)$ is continuous. Take any $s, 0<s \leqq m$. We shall assume $f_{j}(x)$ is continuous for $s<j \leqq m$, if there are such values of $j$, and shall prove that $f_{s}(x)$ is continuous.

Let $x_{0}, \cdots, x_{8}$ be distinct points of $A$. If we subtract (2.6) with $x$ replaced by $x_{0}$ from the same equation with $x$ replaced by $x_{1}$, we find

$$
\begin{aligned}
f_{s}\left(x_{1}\right)-f_{s}\left(x_{0}\right)= & s ! \sum_{j=s+1}^{m}\left[\frac{f_{j}\left(x_{0}\right)}{j !} \sum_{i=0}^{s} \alpha^{i} u_{0 i}^{j}-\frac{f_{j}\left(x_{1}\right)}{j !} \sum_{i=0}^{s} \alpha^{i} u_{1 i}^{j}\right] \\
& +s ! \sum_{i=0}^{\dot{1}} \alpha^{i}\left[R\left(x_{i}, x_{0}\right)-R\left(x_{i}, x_{1}\right)\right] .
\end{aligned}
$$

Given any limit point $x_{0}$ of $A$ and any $\epsilon>0$, take $\delta<\epsilon /\left[2^{s+3}(s+1) m M\right]$ (if $s<m)$ and $<1 / 2$ so small that (3.2) holds with $x$ and $\epsilon$ replaced by $x_{0}$ and $\epsilon /\left[2^{\bullet+2}(s+1) !\right]$ respectively, where $M=\max \left|f_{j}\left(x^{\prime}\right)\right|\left(\left|x^{\prime}-x_{0}\right| \leqq 1, s<j \leqq m\right)$. If $s>1$, take a point $x_{s}$ of $A$ within $\delta$ of $x_{0}$, and take points $x_{s-1}, \cdots, x_{2}$ of $A$ so that $r_{0 i}<r_{0, i+1} / 3(i=2, \cdots, s-1)$. Now take any point $x_{1}$ within $\delta$ 
of $x_{0}$, so that $r_{01}<r_{02} / 3$ if $s>1$. From (13.1) we see that $\left|f_{8}\left(x_{1}\right)-f_{8}\left(x_{0}\right)\right|<\epsilon$, as required (see the proof of Lemma 6).

Let $x_{0}, \cdots, x_{s}$ be an ordered set of points. We say they form an $\left(x_{0}, \rho\right)$-set $(\rho>1)$, if

$$
r_{0, i-1}<\frac{r_{0 i}}{\rho} \quad(i=1, \cdots, s) .
$$

Theorem II. Let $f(x)=f_{0}(x), \cdots, f_{m}(x)$ be defined in the closed set $A$. A necessary condition that a Taylor's expansion for $f(x)$ should hold to the mth order locally uniformly in terms of $f_{0}(x), \cdots, f_{m}(x)$ is that for each (or some) $\rho>1$, each $s(0 \leqq s \leqq m)$, each point $x$ of $A$, and each $\epsilon>0$, there exist $a \delta>0$, such that if $x_{0}, \cdots, x_{s}$ is any $\left(x_{0}, \rho\right)$-set of points lying within $\delta$ of $x$, then

$$
\left|\Delta_{0} \ldots s f-f_{s}(x)\right|<\epsilon .
$$

By the last lemma, the $f_{j}(x)$ are continuous. Take $M$ so that $\left|f_{j}\left(x^{\prime}\right)\right|<M$ for $\left|x^{\prime}-x\right|<1$. Take $\delta<\epsilon(\rho-1)^{s} /\left[2(s+1) m M \rho^{s}\right]$ and $<1$ so that $\mid f_{s}\left(x^{\prime}\right)$ $-f_{s}(x) \mid<\epsilon / 2\left(\left|x^{\prime}-x\right|<\delta\right)$, and so that (3.2) holds with $\epsilon$ replaced by $\epsilon(\rho-1)^{s} /\left[2(s+1) ! \rho^{s}\right]$. Now take any $\left(x_{0}, \rho\right)$-set of points $x_{0}, \cdots, x_{s}$ lying within $\delta$ of $x$. Then

$$
\frac{r_{0 i}}{r_{k i}}<\frac{\rho}{\rho-1}
$$

for $k \neq i$. For if $k<i$, then $r_{0 k} \leqq r_{0, i-1}<r_{0 i} / \rho$, hence $r_{k i} \geqq r_{0 i}-r_{0 k}>r_{0 i}(1-1 / \rho)$, and $r_{0 i} / r_{k i}<1 /(1-1 / \rho)=\rho /(\rho-1)$; if $k>i$, then $r_{0 k} \geqq r_{0, i+1}>\rho r_{0 i}$, hence $r_{k i}$ $\geqq r_{0 k}-r_{0 i}>r_{0 i}(\rho-1)$, and $r_{0 i} / r_{k i}<1 /(\rho-1)<\rho /(\rho-1)$. Replacing $x$ by $x_{0}$ in (2.6) gives immediately $\left|\Delta_{0} \ldots f f-f_{s}\left(x_{0}\right)\right|<\epsilon / 2$; hence $\left|\Delta_{0} \ldots f f-f_{s}(x)\right|<\epsilon$.

THEOREM III. If $A$ is perfect, then the condition in Theorem II is also suffcient.

We shall prove successively for $s=0, \cdots, m$ that $f(x)$ can be expanded in a Taylor's formula to the sth order locally uniformly in terms of $f_{0}(x), \cdots$, $f_{s}(x)$. Evidently $f_{0}(x)$ is continuous; hence this is true for $s=0$. The proof for a general $s$ follows the proof of Lemma 6; we need merely be careful to choose $x_{s-1}, \cdots, x_{1}$ so that $r_{0, t-1}<r_{0 t} / \rho(t=2, \cdots, s)$.

14. Taylor's formula and differentiability. We shall say the set $A$ has the property $Z_{\rho}$ at the point $x(\rho>1)$ if there is an $\eta>0$ such that corresponding to any two points $x_{0}$ and $x_{1}$ of $A$ within $\eta$ of $x$, points $x_{2}, \cdots, x_{s}$ of $A$ can be found such that

$$
\frac{1}{\rho}<\left|\frac{x_{i}-x_{i}}{x_{1}-x_{0}}\right|<\rho \quad(i, j=0, \cdots, s ; i \neq j)
$$


then $r_{i j} / r_{k l}<\rho^{2}$ for $i \neq j, k \neq l$. This condition is satisfied for instance by Cantor's set. $s$ is any number $\leqq m, m$ fixed.

TheOREM IV.* Let $A$ be a closed set having the property $Z_{\rho}$ for some $\rho=\rho(x)$ at each point $x$, and let $f(x)=f_{0}(x), \cdots, f_{m}(x)$ be defined in $A . A$ necessary and sufficient condition that $f(x)$ be of class $C^{m}$ in terms of $f_{0}(x), \cdots, f_{m}(x)$ is that Taylor's formula for $f(x)$ should hold to the mth order locally uniformly in terms of $f_{0}(x), \cdots, f_{m}(x)$.

In short, in this case, Taylor's formula for $f_{0}(x)$ implies Taylor's formula for each $f_{8}(x)$.

The necessity of the condition being trivial, we turn to the sufficiency. By Lemma $10, f_{m}(x)$ is continuous. It remains to prove that for any $s$, $0<s<m, f_{s}(x)$ may be expanded in a Taylor's formula to the $(m-s)$ th order locally uniformly in terms of $f_{s}(x), \cdots, f_{m}(x)$. We shall prove this for $s$, assuming it for numbers $s+1, \cdots, m$.

Let $x_{0}, \cdots, x_{\varepsilon}$ be distinct points of $A$. Set

$$
\begin{aligned}
H_{i} & =\sum_{i=0}^{s} \alpha^{i} u_{0 i}^{i} \\
H_{i}^{\prime} & =\sum_{i=0}^{\dot{\alpha}} \alpha^{i} u_{1 i}^{j}=\sum_{i=0}^{s} \alpha^{i}\left(u_{0 i}-u_{01}\right)^{i}=\sum_{i=0}^{\infty} \alpha^{i} \sum_{l}(-1)^{i-l}\left(\begin{array}{l}
j \\
l
\end{array}\right) u_{0 i}^{l} u_{01}^{j-l} \\
& =\sum_{l}(-1)^{i-l}\left(\begin{array}{l}
j \\
l
\end{array}\right) H_{l} u_{01}^{j-l},
\end{aligned}
$$

where $\sum_{l}$ means summation over all values of $l$. We can write (if $s<m$ )

$$
\begin{aligned}
\sum_{j=++1}^{m} \frac{f_{j}\left(x_{1}\right)}{j !} H_{l}^{\prime} & =\sum_{j=-+1}^{m} \frac{1}{j !} \sum_{k=i}^{m} \frac{f_{k}\left(x_{0}\right)}{(k-j) !} u_{01}^{k-i} \sum_{l}(-1)^{i-l}\left(\begin{array}{l}
j \\
l
\end{array}\right) H_{l} u_{01}^{j-l}+R \\
& =\sum_{k=-+1}^{m} \frac{f_{k}\left(x_{0}\right)}{k !} \sum_{l} u_{01}^{k-l} H_{l} \sum_{j=s+1}^{k}(-1)^{j-l}\left(\begin{array}{l}
k \\
j
\end{array}\right)\left(\begin{array}{l}
j \\
l
\end{array}\right)+R,
\end{aligned}
$$

where

$$
R=\sum_{j=s+1}^{m} \frac{1}{j !} H_{j}^{\prime} R_{j}\left(x_{1}, x_{0}\right) .
$$

Now if $k \geqq l>s$, then on replacing $j$ by $k-j$ we find

* For the special case that $A$ is a closed interval, see a paper by the author, Derivatives, difference quotients and Taylor's formula, Bulletin of the American Mathematical Society, vol. 40 (1934), pp. 89-94; Theorem III. 


$$
\begin{aligned}
\sum_{j=s+1}^{k}(-1)^{j-l}\left(\begin{array}{l}
k \\
j
\end{array}\right)\left(\begin{array}{l}
j \\
l
\end{array}\right) & =\sum_{j}(-1)^{k-j-l}\left(\begin{array}{l}
k \\
j
\end{array}\right)\left(\begin{array}{c}
k-j \\
l
\end{array}\right) \\
& =(-1)^{k-l}\left(\begin{array}{c}
0 \\
k-l
\end{array}\right)=\delta_{k l},{ }^{*}
\end{aligned}
$$

and if $k>l=s$,

$$
\sum_{j=s+1}^{k} \cdot(-1)^{j-l}\left(\begin{array}{l}
k \\
j
\end{array}\right)\left(\begin{array}{l}
j \\
l
\end{array}\right)=\delta_{k s}-(-1)^{s-s}\left(\begin{array}{l}
k \\
s
\end{array}\right)\left(\begin{array}{l}
s \\
s
\end{array}\right)=-\left(\begin{array}{l}
k \\
s
\end{array}\right) .
$$

Therefore, as $H_{l}=0(l<s)$ and $H_{s}=1$,

$$
\sum_{j=s+1}^{m} \frac{f_{j}\left(x_{1}\right)}{j !} H_{j}^{\prime}=\sum_{k=s+1}^{m} \frac{f_{k}\left(x_{0}\right)}{k !}\left[-\left(\begin{array}{l}
k \\
s
\end{array}\right) u_{01}^{k-s}+H_{k}\right]+R .
$$

Putting this in (13.1) gives

$$
\begin{aligned}
f_{s}\left(x_{1}\right)= & \sum_{k=s}^{m} \frac{f_{k}\left(x_{0}\right)}{(k-s) !} u_{01}^{k-s}-\sum_{j=s+1}^{m} \frac{s !}{j !} \sum_{i=0}^{s} \alpha^{i} u_{1 i}^{i} R_{j}\left(x_{1}, x_{0}\right) \\
& +s ! \sum_{i=0}^{s} \alpha^{i}\left[R\left(x_{i}, x_{0}\right)-R\left(x_{i}, x_{1}\right)\right] .
\end{aligned}
$$

Given a point $x$ of $A$ and an $\epsilon>0$, take $\rho$ and $\eta$ corresponding to $x$, and take $\delta^{\prime}<\eta$ so that (3.2) holds with $\delta$ and $\epsilon$ replaced by $\delta^{\prime}$ and $\epsilon /\left[3 m(s+1) ! \rho^{2 m}\right]$ and with $s$ taking on the values $0, s+1, \cdots, m$. Set $\delta=\delta^{\prime} /(2 \rho)$. Now if $x_{0}$ and $x_{1}$ are points of $A$ within $\delta$ of $x$, we can add points $x_{2}, \cdots, x_{8}$ of $A$ so that (14.1) holds and these points will lie within $\delta^{\prime}$ of $x$. Then

$$
\left|\frac{\alpha^{2} u_{1 i}^{\prime}}{u_{01}^{m-s}} R_{j}\left(x_{1}, x_{0}\right)\right|=\frac{r_{1 i}^{j}}{r_{0 i} \cdots r_{i-1, i} r_{i+1, i} \cdots r_{s i} r_{01}^{j-s}} \frac{\left|R_{j}\left(x_{1}, x_{0}\right)\right|}{r_{01}^{m-j}}<\frac{\epsilon}{3 m(s+1) !},
$$

and similarly for the other remainder terms. Therefore $\left|R_{s}\left(x_{1}, x_{0}\right)\right| / r_{01}^{m-s}<\epsilon$, as required.

COROLlaRy. If $m \leqq 2$, Theorem IV holds for all closed sets.

The only value of $s$ we may need in the above proof is $s=1$; the condition $Z_{\rho}$ is satisfied trivially if $s=1$.

Example. Theorem IV does not hold for all closed sets, as we now show, using $m=3$. Set $a_{i}=1 / 2^{i}, \quad b_{i}=1 / 2^{2 i}, \quad c_{i}=1 / 2^{3 i} ; \quad b_{i}^{\prime}=a_{i}+b_{i}, \quad c_{i}^{\prime}=a_{i}+c_{i}$, $d_{i}=a_{i}+b_{i}-c_{i}(i=1,2, \cdots)$. Let $A$ be the set of points $0, a_{i}, c_{i}^{\prime}, d_{i}, b_{i}^{\prime}$. Set $f_{0}(0)=f_{1}(0)=f_{2}(0)=f_{3}(0)=0$,

* See Netto, Lehrbuch der Combinatorik, Leipzig, 1927, \$158, (27). 


$$
\begin{array}{llll}
f_{0}\left(a_{i}\right)=0, & f_{0}\left(c_{i}^{\prime}\right)=0, & f_{0}\left(d_{i}\right)=0, & f_{0}\left(b_{i}^{\prime}\right)=b_{i}{ }^{2} c_{i}, \\
f_{1}\left(a_{i}\right)=0, & f_{1}\left(c_{i}^{\prime}\right)=0, & f_{1}\left(d_{i}\right)=b_{i}{ }^{2}-b_{i} c_{i}, & f_{1}\left(b_{i}^{\prime}\right)=b_{i}{ }^{2}+b_{i} c_{i}, \\
f_{2}\left(a_{i}\right)=0, & f_{2}\left(c_{i}^{\prime}\right)=0, & f_{2}\left(d_{i}\right)=2 b_{i}, & f_{2}\left(b_{i}^{\prime}\right)=2 b_{i}, \\
f_{3}\left(a_{i}\right)=0, & f_{3}\left(c_{i}^{\prime}\right)=0, & f_{3}\left(d_{i}\right)=0, & f_{3}\left(b_{i}^{\prime}\right)=0 .
\end{array}
$$

As $\Delta\left(0, a_{i}, c_{i}^{\prime}, d_{i}\right)=0$, while $\Delta\left(a_{i}, c_{i}^{\prime}, d_{i}, b_{i}^{\prime}\right)=3 ! b_{i}{ }^{2} c_{i} /\left[b_{i}\left(b_{i}-c_{i}\right) c_{i}\right] \rightarrow 6$ as $i \rightarrow \infty, \Delta^{3} f_{0}(x)$ does not converge at $x=0$, and hence $f_{0}(x)$ is not of class $C^{3}$, by Theorem I. However, Taylor's formula holds for $f_{0}(x)$ to the third order locally uniformly. For a calculation shows that $R(x, y)=0$ whenever $x$ and $y$ are chosen from the points $a_{i}, c_{i}^{\prime}, d_{i}, b_{i}^{\prime}$, except that $R\left(b_{i}^{\prime}, a_{i}\right)=R\left(b_{i}^{\prime}, c_{i}^{\prime}\right)=b_{i}{ }^{2} c_{i}$, $R\left(c_{i}^{\prime}, d_{i}\right)=R\left(c_{i}^{\prime}, b_{i}^{\prime}\right)=b_{i} c_{i}\left(b_{i}-2 c_{i}\right)$; hence if $x$ and $y$ are chosen in any manner from the points $a_{i}, c_{i}^{\prime}, d_{i}, b_{i}^{\prime}, R(y, x) /(y-x)^{3} \rightarrow 0$ as $i \rightarrow \infty$. Suppose now $x_{i}$ and $y_{j}$ are chosen from $a_{i}, c_{i}^{\prime}, d_{i}, b_{i}^{\prime}$, and from $a_{j}, c_{j}^{\prime}, d_{j}, b_{j}^{\prime}$ respectively, $j \neq i$ (or $x_{i}=0$ or $\left.y_{j}=0\right)$. If $k$ is the larger of the numbers $i, j$, then

$$
\left|R\left(y_{j}, x_{i}\right)\right|<2 b_{k}^{2} c_{k}+\left(b_{k}^{2}+b_{k} c_{k}\right)\left(a_{k}+b_{k}\right)+b_{k}\left(a_{k}+b_{k}\right)^{2},
$$

and as $\left|y_{i}-x_{i}\right|^{3} \geqq a_{k}{ }^{3} / 8, R\left(y_{j}, x_{i}\right) /\left(y_{j}-x_{i}\right)^{3} \rightarrow 0$ as $i, j \rightarrow \infty(j \neq i)$. Hence for some $\delta>0$, if $x$ and $y$ are any two points of $A$ within $\delta$ of $0,\left|R(y, x) /(y-x)^{3}\right|$ $<\epsilon$. This is true also at each isolated point of $A$; hence Taylor's formula is valid.

Note that we may increase $A$ to a perfect set by adding the intervals between $a_{i}$ and $c_{i}^{\prime}$ and between $d_{i}$ and $b_{i}^{\prime}$, and giving the obvious definitions of $f_{0}(x), \cdots, f_{3}(x)$ there. In this example, Taylor's formula holds to the required order for neither $f_{1}(x)$ nor $f_{2}(x)$.

\section{HARVARD UNIVERSITY,}

Cambridge, Mass. 